\title{
Análise comparativa da satisfação do cardápio e aceitabilidade de preparações vegetarianas em restaurante universitário
}

\author{
Comparative analysis of satisfaction of the menu and acceptability of vegetarian preparations in a \\ university restaurant
}

Análisis comparativo de satisfacción del menu y aceptabilidad de preparados vegetarios en restaurante Universitario

Recebido: 01/09/2021 | Revisado: 08/09/2021 | Aceito: 10/09/2021 | Publicado: 13/09/2021

João Felipe de Almeida Fonseca

ORCID: https://orcid.org/0000-0002-0012-7437 Universidade Federal do Espírito Santo, Brasil E-mail: jfaf18@gmail.com

Michelly de Araújo Marques

ORCID: https://orcid.org/0000-0003-1761-9398 Universidade Federal do Espírito Santo, Brasil E-mail: michellymarqss@gmail.com

Lívia Bollis Campagnaro

ORCID: https://orcid.org/0000-0003-2314-4894 Universidade Federal do Espírito Santo, Brasil E-mail: liviabollisc@gmail.com

Loise Nascimento dos Santos

ORCID: https://orcid.org/0000-0002-0512-8885

Universidade Federal do Espírito Santo, Brasil E-mail: loise_98@hotmail.com

Leticia Rangel Pereira

ORCID: https://orcid.org/0000-0003-0235-1317 Universidade Federal do Espírito Santo, Brasil E-mail: leticiarangelpe@gmai.com

Oscar Geovanny Enriquez Martinez ORCID: https://orcid.org/0000-0003-4561-122X Universidade Federal do Espírito Santo, Brasil E-mail: ndoscar.enriquez@gmai.com

Daniela Alves Silva

ORCID: https://orcid.org/0000-0001-7396-2305

Universidade Federal do Espírito Santo, Brasil E-mail: contato.daniela.nut@gmai.com

\begin{abstract}
Resumo
Objetivo: avaliar a satisfação do cardápio e comparar a aceitabilidade de preparações vegetarianas entre vegetarianos e onívoros. Metodologia: Estudo transversal realizado em dois RU de Vitória-ES (campus 1 e 2). A coleta de dados ocorreu por meio da aplicação de questionário para identificação da aceitabilidade dos pratos vegetarianos, além da mensuração de resto-ingestão. Os dados foram analisados no SPSS, adotando-se p<0,05. Resultados: Identificou-se que 19,2\% dos participantes eram vegetarianos, os quais apresentaram mediana da idade semelhante aos onívoros (22 anos). A maioria era do sexo feminino (53,9\% dos onívoros e 68,2\% dos vegetarianos), que se mostrou associado ao vegetarianismo $(\mathrm{p}<0,001)$. O Quibe Vegetariano foi o único prato que apresentou diferença das notas de aceitabilidade, sendo as maiores atribuídas pelos onívoros. A preparação de menor aceitabilidade foi a Torta de Soja. A satisfação do cardápio foi baixa em ambos os campi, visto elevados valores de resto-ingestão. Conclusão: Informações sobre satisfação do cardápio e perfil dos clientes de unidades de alimentação e nutrição são importantes no atendimento satisfatório do público e na promoção de boas práticas ambientais.
\end{abstract}

Palavras-chave: Vegetarianismo; Desperdício de alimentos; Satisfação do usuário.

\begin{abstract}
Objective: Evaluate menu satisfaction and compare the acceptability of vegetarian preparations between vegetarians and omnivores. Methodology: Cross-sectional study carried out in two UR in Vitória-ES (campus 1 and 2). Data collection occurred through the application of a questionnaire to identify the acceptability of vegetarian dishes, in addition to measuring rest-intake. The data were analyzed in SPSS, adopting $p<0.05$. Results: $19.2 \%$ of the participants were vegetarians, who had a median age similar to omnivores ( 22 years old). The majority were female (53.9\% of omnivores and $68.2 \%$ of vegetarians), which was associated with vegetarianism $(\mathrm{p}<0.001)$. Vegetarian
\end{abstract}


Kibbeh was the only dish that showed a difference in acceptability scores, the largest being atributed by omnivores. The least acceptable preparation was the Soy Pie. Menu satisfaction was low on both campuses, given high levels of rest-intake. Conclusion: Information on the satisfaction of the menu and the profile of the customers of food and nutrition units are important in satisfying public service and in promoting good environmental practices.

Keywords: Vegetarianism; Food waste; User satisfaction.

\section{Resumen}

Objetivo: Evaluar la satisfacción del menú y comparar la aceptabilidad de las preparaciones vegetarianas entre vegetarianos y omnívoros. Metodología: Estudio transversal realizado en dos RU de Vitória-ES (campus 1 y 2). La recolección de datos se realizó mediante la aplicación de un cuestionario para identificar la aceptabilidad de platos vegetarianos, además de la medición de la ingesta sobrante. Los datos se analizaron mediante SPSS, adoptando p<0,005. Resultados: Se identificó que el 19,2\% de los participantes eran vegetarianos, quienes tenían una mediana de edad similar a la de los omnívoros (22 años). La mayoría eran mujeres (53,9\% de omnívoros y 68,2\% de vegetarianos), lo que se asoció con el vegetarianismo ( $\mathrm{p}<0,001)$. El Quibe Vegetariano fue el único platillo que presentó diferencia en los grados de aceptabilidad, siendo los más altos atribuidos por omnívoros. La preparación menos aceptable fue em pastel de soja. La satisfacción del menú fue baja en ambos campus, ya que los valores de ingesta de descanso fueron altos. Conclusión: La información sobre la satisfacción del menú y el perfil de los clientes en las unidades de alimentación y nutrición son importantes para brindar un servicio satisfactorio al público y promover las buenas prácticas ambientales.

Palabras clave: Vegetarianismo; Desechos alimentarios; Satisfacción del usuario.

\section{Introdução}

A alimentação fora do âmbito domiciliar está cada vez mais prevalente na rotina da população e segundo os dados da última Pesquisa de Orçamento Familiar, tal prática correspondeu à 32,8\% das despesas dos brasileiros com alimentação (Instituto Brasileiro de Geografia e Estatística, 2019). Como forma de suprir essa demanda crescente, o mercado de alimentação coletiva integra vários serviços, de natureza comercial e institucional, representados pelas Unidades de Alimentação e Nutrição (UANs) (Associação Brasileira das Empresas de Refeições Coletivas, 2008, Brito, \& Oliveira, 2017, Brasil, 2018)

No ambiente universitário, ações relacionadas à alimentação são parte da assistência estudantil preconizada pelo Programa Nacional de Assistência Estudantil (PNAES), criado em 2010 com o objetivo de ampliar as condições de permanência dos jovens na educação superior pública (Brasil, 2010). Neste ambiente, os Restaurantes Universitários (RU) são exemplos de UAN que atendem um público diversificado e heterogêneo, buscando fornecer, majoritariamente, refeições balanceadas e atrativas a um preço acessível, segundo as necessidades alimentares e nutricionais dessa coletividade (Benvindo, Pinto \& Bandoni, 2017).

Práticas alimentares como o vegetarianismo têm sido identificadas como demandas de atendimento em RU e, ao mesmo tempo em que se apresentam como desafio para o serviço, tornam-se oportunidades para o cumprimento do direito humano à alimentação adequada, principalmente aos adeptos a esse estilo de vida por motivos éticos ou ambientais que podem não se atentar às possíveis deficiências nutricionais (Leitzmann, 2014, Sociedade Vegetariana Brasileira, 2016; Barros, Bierhals, \& Assunção, 2020). A alimentação vegetariana diz respeito à restrição total ou parcial do consumo de produtos de origem animal e pode ser classificada de acordo com suas características em semivegetariano, pescovegetariano, ovolactovegetariano, vegetariano estrito e vegano (Tonstad et al. 2013).

Ressalta-se que informações acerca do perfil dos usuários como o estado nutricional, hábitos alimentares, aceitabilidade das preparações e do cardápio e a satisfação com o serviço, são fundamentais para o atendimento adequado (Benvindo, Pinto \& Bandoni, 2017, Brito, \& Oliveira, 2017). Além disso, o desperdício de alimentos é um indicador de grande relevância e pode apontar baixa qualidade da refeição, sendo primordial controlar, comparar e avaliar os processos de produção. Assim, será possível ofertar refeições atrativas para os clientes e adotar medidas que agreguem mudanças 
comportamentais e, consequentemente, a conscientização dos comensais e dos manipuladores dos alimentos para evitar o excesso de produção, sobras e restos (Bacalhau \& Ferreira, 2017).

O presente estudo tem como objetivo avaliar a satisfação do cardápio e comparar a aceitabilidade de preparações vegetarianas entre clientes vegetarianos e onívoros de restaurantes universitários.

\section{Metodologia}

Compreende-se um estudo de caráter transversal que foi realizado em dois RUs de uma instituição de ensino superior do município de Vitória - ES. O RU central produz e distribui refeições para dois campi da instituição de ensino e está localizado no campus que concentra os cursos de diferentes áreas de conhecimento, exceto saúde, e atende tanto os estudantes destes cursos nos serviços de almoço e jantar (refeição in loco) quanto os estudantes do campus da área da saúde no serviço almoço (refeição transportada). Neste estudo, a unidade central será denominada campus 1 e a unidade que atende os estudantes da área da saúde, campus 2.

A coleta de dados foi realizada nos próprios RUs durante os meses de outubro e novembro de 2019. Foram incluídos indivíduos acima de 18 anos de idade que manifestaram interesse em participar e assinaram o Termo de Consentimento Livre e Esclarecido (TCLE). Foram excluídos aqueles que não responderam a entrevista completa de acordo com o seu perfil (onívoro/vegetariano).

Com intuito de conhecer o público que consumia a preparação vegetariana dos RUs e identificar os adeptos ao vegetarianismo, os clientes que optaram por consumir esse prato nos dias de coleta de dados receberam um informativo contendo o objetivo do estudo e convite para participação. Os mesmos foram abordados na saída dos restaurantes e, após registro do consentimento, responderam um breve questionário contendo perguntas de caracterização sociodemográfica, frequência de consumo e aceitabilidade do prato vegetariano do dia e de adesão à alimentação vegetariana. Os participantes que se autodeclararam vegetarianos responderam outro questionário semiestruturado sobre a prática do vegetarianismo contendo perguntas sobre o consumo ou não de produtos de origem animal e tempo de adesão. Cabe ressaltar que a participação no estudo foi única, ou seja, mesmo que o cliente tivesse consumido a preparação vegetariana durante vários dias de coleta, o mesmo respondeu o questionário da pesquisa somente uma vez.

A aceitabilidade das preparações vegetarianas foi avaliada por meio de escala do tipo likert de 5 pontos, sendo 1 equivalente a desgostei muitíssimo e 5, a gostei muitíssimo, para os atributos de sabor, aroma, textura, aparência e aceitação geral (Minim, 2006). O índice de aceitabilidade (IA) para cada atributo foi calculado pela fórmula: IA (\%) = A x 100 / B, onde, $\mathrm{A}=$ nota média obtida para o produto e $\mathrm{B}=$ nota máxima dada ao produto (Dutcosky, 1996, Dutcosky, 2011). Ressalta-se que neste estudo foram analisadas as mesmas preparações que foram servidas tanto no campus 1 quanto no campus 2 durante a coleta, totalizando cinco preparações.

A satisfação do cardápio foi investigada por meio do desperdício de alimentos avaliado em 17 dias (11 no campus 1 e 6 no campus 2) a partir da pesagem do resto ingestão (RI) em balança plataforma da marca Balmak®, capacidade máxima de $500 \mathrm{Kg}$ e precisão de $100 \mathrm{~g}$. Os dias de coleta do desperdício foi maior no campus 1 por este atender uma maior quantidade de clientes. O RI consiste nos alimentos devolvidos no prato pelo cliente logo após a realização da sua refeição, separando-se os ossos e cascas (Vaz, 2006). A informação do número de clientes atendidos foi coletada para estimar o RI por cliente, obtido pela divisão do peso total do resto (em kg) pelo número de clientes e o resultado convertido em gramas. A quantificação e classificação foram realizadas conforme recomendação de Vaz (2006), considerando-se adequado os valores inferiores a $30 \mathrm{~g}$ por cliente.

Os dados foram armazenados no Microsoft Excel® e analisados no programa SPSS (Statistical Package for the Social Sciences $\left.{ }^{\circledR}\right)$, versão 20.0. Os resultados obtidos foram apresentados na forma de estatística descritiva com dados em frequência 
(\%), média, desvio-padrão e mediana. Foram realizados testes de associação (qui-quadrado) e de comparação de grupos. Para testar a normalidade das variáveis numéricas foi aplicado o teste Kolmogorov-Smirnov, seguido do teste deMann-Whitney. O nível de significância adotado foi de $\mathrm{p}<0,05$.

O estudo foi aprovado pelo Comitê de Ética em Pesquisa com Seres Humanos da Universidade Federal do Espírito Santo (parecer $\mathrm{n}^{\mathrm{o}}$ 3.445.226 e CAAE 14084019.2.0000.5060). A identidade e dados pessoais dos participantes foram preservados com a garantia de sigilo absoluto.

\section{Resultados}

Foram entrevistados um total de 1387 clientes, sendo 80,2\% (n=1113) onívoros e 19,8\% (n=274) vegetarianos. A caracterização do público, segundo prática vegetariana e campi, está disposta na Tabela 1.

A mediana (mínimo-máximo) de idade dos onívoros foi de 22 (18-80) anos e de 22 (18-64) para os vegetarianos, sem diferença estatística entre esses grupos $(\mathrm{p}=0,46)$, bem como entre os campi $(\mathrm{p}=0,23$ para os onívoros e $\mathrm{p}=0,22$ para os vegetarianos) (Tabela 1).

Observou-se que a prática do vegetarianismo esteve associada ao sexo feminino, este em maior proporção na população estudada $(53,9 \%$ dos onívoros e $68,2 \%$ dos vegetarianos, sendo p<0,001). Mais de $95 \%$ dos entrevistados eram estudantes independentemente da prática alimentar $(\mathrm{p}=0,37)$ e dos campi de estudo $(\mathrm{p}=0,80$ entre onívoros e $\mathrm{p}=0,97$ entre vegetarianos) (Tabela 1).

No que concerne à frequência de consumo da opção vegetariana, o consumo diário foi maior entre o total de vegetarianos $(49,6 \% ; n=137)$ em comparação com os onívoros $(7,6 \%, n=85)$, com diferença estatisticamente significante ( $\mathrm{p}<0,001)$. Quando comparados entre os campi, foi observado maior consumo pelos onívoros do campus 2 ( $\mathrm{p}=0,001$ ), conforme Tabela 1. 
Tabela 1 - Caracterização de clientes de acordo com os campi do restaurante universitário. Vitória, ES, 2019.

\begin{tabular}{|c|c|c|c|c|c|c|c|}
\hline \multirow[b]{2}{*}{ Características } & \multicolumn{3}{|c|}{ Onívoros $(\mathrm{n}=1113-80,2 \%)$} & \multicolumn{4}{|c|}{ Vegetarianos $(n=274-19,8 \%)$} \\
\hline & Total & Campus 1 & Campus 2 & Total & Campus 1 & Campus 2 & $\begin{array}{l}\text { Valor } \\
\text { de } p^{c}\end{array}$ \\
\hline \multicolumn{8}{|l|}{ Idade em anos $^{\mathrm{a}}$} \\
\hline $\mathrm{Med} \pm \mathrm{DP}$ & $24,1 \pm 7,3$ & $23,29 \pm 5,9$ & $24,43 \pm 7,8$ & $23,63 \pm 6,4$ & $22,43 \pm 4,5$ & $23,9 \pm 6,7$ & \\
\hline $\operatorname{Md}(\min -\max )$ & $\begin{array}{l}22(18- \\
80)\end{array}$ & $\begin{array}{l}22(18- \\
70)\end{array}$ & $\begin{array}{l}22(18- \\
80)\end{array}$ & $\begin{array}{l}22(18- \\
64)\end{array}$ & $\begin{array}{l}21(18- \\
43)\end{array}$ & $\begin{array}{l}22(18- \\
64)\end{array}$ & 0,46 \\
\hline Valor de $\mathrm{p}^{\mathrm{d}}$ & 0,23 & & & 0,22 & & & \\
\hline \multicolumn{8}{|l|}{$\operatorname{Sexo}, \%(n)^{b}$} \\
\hline Feminino & $53,9(600)$ & $71,7(233)$ & $46,6(367)$ & $68,2(187)$ & $78,4(40)$ & $65,9(147)$ & \\
\hline Masculino & $46,1(513)$ & $28,3(98)$ & $53,4(421)$ & $31,8(274)$ & $21,6(11)$ & $34,1(76)$ & $<0,001$ \\
\hline Valor de $\mathrm{p}^{\mathrm{d}}$ & $<0,001$ & & & 0,08 & & & \\
\hline \multicolumn{8}{|l|}{ Profissão, \% (n) ${ }^{\mathbf{b}}$} \\
\hline Estudante & $\begin{array}{l}97,0 \\
(1081)\end{array}$ & $97,2(316)$ & $97,0(765)$ & $95,7(264)$ & $96,1(49)$ & $95,6(215)$ & 0,37 \\
\hline Outros & $3,0(33)$ & $2,8(9)$ & $3,0(24)$ & $4,0(11)$ & $3,9(2)$ & $4,0(9)$ & \\
\hline Valor de $\mathrm{p}^{\mathrm{d}}$ & 0,80 & & & 0,97 & & & \\
\hline $\begin{array}{l}\text { Frequência } \\
\text { consumo da ops } \\
\text { vegetariana, } \%(n)\end{array}$ & & & & & & & \\
\hline Esporadicamente & $27,8(310)$ & $28(91)$ & 27,8 (219) & $7,2(20)$ & $9,8(5)$ & $6,7(15)$ & \\
\hline 1 a 2 vezes/semana & $32,2(359)$ & $38,8(126)$ & $29,5(233)$ & $12,3(34)$ & $11,8(6)$ & $12,4(28)$ & \\
\hline 3 a 4 vezes/semana & 32,2 (359) & 28,9 (94) & $33,6(265)$ & $30,1(83)$ & $33,3(17)$ & $29,3(66)$ & \\
\hline Diariamente & $7,6(85)$ & $4,0(13)$ & $9,1(72)$ & $49,6(137)$ & $43,1(22)$ & $51,1(115)$ & $<0,001$ \\
\hline Valor de $\mathrm{p}^{\mathrm{d}}$ & 0,001 & & & 0,72 & & & \\
\hline
\end{tabular}

Med: Média; DP: Desvio-padrão; Md: Mediana; a: Teste de Mann-Whitney; b: Teste de Qui-Quadrado; c: Comparação entre vegetarianos e onívoros; ${ }^{\mathrm{d}}$. Comparação entre campus 1 e campus 2. Fonte: Autores.

Quanto aos vegetarianos, identificou-se predomínio da dieta ovolactovegetariana $(80,4 \%, \mathrm{n}=222)$, sem diferença entre os campi $(\mathrm{p}=0,47)$. A mediana do tempo de prática vegetariana foi de 24 meses, variando entre 1 e 600 meses, não diferindo entre os campi $(\mathrm{p}=0,51)$ (Tabela 2$)$.

Tabela 2 - Caracterização da prática vegetariana dos adeptos participantes do estudo, segundo campi. Vitória, ES, 2019.

\begin{tabular}{lllll}
\hline Informações & Total & Campus 1 & Campus 2 & Valor de p \\
\hline Tipo de vegetarianismo, \% (n) $\mathbf{a}^{\mathbf{a}}$ & & & & \\
Semivegetariano & $4,0(11)$ & $5,9(3)$ & $3,6(8)$ & \\
Pescovegetariano & $8,7(24)$ & $7,8(4)$ & $8,9(20)$ & \\
Ovolactovegetariano & $80,4(222)$ & $76,5(39)$ & $81,3(183)$ & 0,47 \\
Vegetariano Estrito & $2,5(7)$ & $5,9(3)$ & $1,8(4)$ & \\
Vegano & $4,3(12)$ & $3,9(2)$ & $4,4(10)$ & \\
Tempo de prática vegetariana em & & & & \\
meses $^{\mathbf{b}}$ & & & & \\
Med \pm DP & $49,1 \pm 73,5$ & $42,6 \pm 59,9$ & $50,5 \pm 76,4$ & \\
Md (min - max) & $24(1-600)$ & $24(1-305)$ & $24(1-600)$ & 0,51 \\
\hline
\end{tabular}

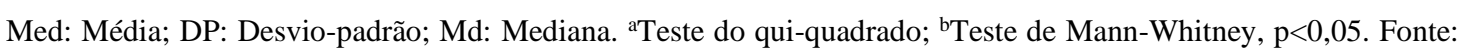
Autores. 
Observou-se que a mediana de notas mais identificada para os diferentes atributos das preparações avaliadas foi de 4,00, a qual corresponde a categoria denominada gostei. Porém, para os atributos aroma e/ou sabor de alguns pratos encontrouse mediana igual a 3,00, correspondente à indiferente. A única preparação que apresentou diferença nas notas foi o Quibe Vegetariano sendo melhor avaliada pelos onívoros (Tabela 3).

Tabela 3 - Distribuição das notas das preparações vegetarianas fornecidas nos campi de um restaurante universitário, de acordo com a adesão à prática. Vitória, ES, 2019.

\begin{tabular}{|c|c|c|c|c|c|c|}
\hline $\begin{array}{l}\text { Preparação } \\
\text { Vegetariana }\end{array}$ & n & Sabor & Aroma & Textura & Aparência & Aceitação Geral \\
\hline & \multicolumn{6}{|c|}{$\begin{array}{l}\text { Media+DP/Md } \\
(\min -\max )\end{array}$} \\
\hline \multicolumn{7}{|l|}{$\begin{array}{l}\text { Escondidinho } \\
\text { de Cará com } \\
\text { PTS }\end{array}$} \\
\hline Onívoros & $\begin{array}{c}8 \\
0\end{array}$ & $\begin{array}{l}3,9 \pm 0,9 / 4,00 \\
(1-5)\end{array}$ & $\begin{array}{l}3,4 \pm 1,0 / 4,00 \\
(1-5)\end{array}$ & $\begin{array}{l}3,7 \pm 1,0 / 4,00 \\
(1-5)\end{array}$ & $\begin{array}{l}3,9 \pm 0,9 / 4,00 \\
(1-5)\end{array}$ & $\begin{array}{l}3,7 \pm 1,0 / 4,00 \\
(1-5)\end{array}$ \\
\hline Vegetarianos & $\begin{array}{c}2 \\
0\end{array}$ & $\begin{array}{l}3,9 \pm 0,9 / 4,00 \\
(2-5)\end{array}$ & $\begin{array}{l}3,5 \pm 1,0 / 3,50 \\
(2-5)\end{array}$ & $\begin{array}{l}3,8 \pm 1,2 / 4,00 \\
(1-5)\end{array}$ & $\begin{array}{l}3,7 \pm 1,4 / 4,00 \\
(1-5)\end{array}$ & $\begin{array}{l}3,9 \pm 0,8 / 4,00 \\
(2-5)\end{array}$ \\
\hline \multicolumn{7}{|l|}{ Falafel } \\
\hline Onívoros & $\begin{array}{l}3 \\
1\end{array}$ & $\begin{array}{l}4,2 \pm 0,6 / 4,00 \\
(3-5)\end{array}$ & $\begin{array}{l}3,5 \pm 0,9 / 3,00 \\
(1-5)\end{array}$ & $\begin{array}{l}3,9 \pm 0,9 / 4,00 \\
(2-5)\end{array}$ & $\begin{array}{l}4,1 \pm 1,0 / 4,00 \\
(2-5)\end{array}$ & $\begin{array}{l}4,1 \pm 0,6 / 4,00 \\
(3-5)\end{array}$ \\
\hline Vegetarianos & 9 & $\begin{array}{l}3,5 \pm 1,4 / 4,00 \\
(1-5)\end{array}$ & $\begin{array}{l}3,5 \pm 1,3 / 4,00 \\
(1-5)\end{array}$ & $\begin{array}{l}4,2 \pm 0,8 / 4,00 \\
(3-5)\end{array}$ & $\begin{array}{l}3,7 \pm 1,2 / 4,00 \\
(1-5)\end{array}$ & $\begin{array}{l}3,6 \pm 1,1 / 4,00 \\
(1-5)\end{array}$ \\
\hline $\begin{array}{l}\text { Valor de } \mathrm{p}^{\mathrm{a}} \\
\text { Polenta com } \\
\text { Lentilha }\end{array}$ & - & 0,276 & 0,750 & 0,545 & 0,444 & 0,406 \\
\hline Onívoros & $\begin{array}{l}1 \\
4 \\
3\end{array}$ & $\begin{array}{l}3,9 \pm 0,9 / 4,00 \\
(1-5)\end{array}$ & $\begin{array}{l}3,6 \pm 1,0 / 4,00 \\
(1-5)\end{array}$ & $\begin{array}{l}3,6 \pm 1,1 / 4,00 \\
(1-5)\end{array}$ & $\begin{array}{l}3,3 \pm 1,2 / 3,00 \\
(1-5)\end{array}$ & $\begin{array}{l}3,8 \pm 0,84,00 \\
(1-5)\end{array}$ \\
\hline Vegetarianos & $\begin{array}{l}3 \\
9\end{array}$ & $\begin{array}{l}3,9 \pm 0,9 / 4,00 \\
(1-5)\end{array}$ & $\begin{array}{l}3,4 \pm 1,0 / 3,00 \\
(1-5)\end{array}$ & $\begin{array}{l}3,3 \pm 1,1 / 4,00 \\
(1-5)\end{array}$ & $\begin{array}{l}3,1 \pm 1,2 / 3,00 \\
(1-5)\end{array}$ & $\begin{array}{l}3,7 \pm 0,9 / 4,00 \\
(1-5)\end{array}$ \\
\hline $\begin{array}{l}\text { Valor de } \mathrm{p}^{\mathrm{a}} \\
\text { Quibe } \\
\text { Vegetariano }\end{array}$ & - & 0,991 & 0,294 & 0,072 & 0,468 & 0,468 \\
\hline Onívoros & $\begin{array}{l}3 \\
3 \\
0\end{array}$ & $\begin{array}{l}4,2 \pm 0,8 / 4,0 \\
(1-5)\end{array}$ & $\begin{array}{l}3,7 \pm 1,0 / 4,0 \\
(1-5)\end{array}$ & $\begin{array}{l}3,8 \pm 1,0 / 4,0 \\
(1-5)\end{array}$ & $\begin{array}{l}4,0 \pm 0,9 / 4,0 \\
(1-5)\end{array}$ & $\begin{array}{l}4,1 \pm 0,8 / 4,0 \\
(1-5)\end{array}$ \\
\hline Vegetarianos & $\begin{array}{c}6 \\
5\end{array}$ & $\begin{array}{l}4,0 \pm 0,9 / 4,0 \\
(1-5)\end{array}$ & $\begin{array}{l}3,5 \pm 0,9 / 3,0 \\
(2-5)\end{array}$ & $\begin{array}{l}3,5 \pm 1,1 / 4,0 \\
(1-5)\end{array}$ & $\begin{array}{l}3,8 \pm 1,0 / 4,0 \\
(1-5)\end{array}$ & $\begin{array}{l}3,8 \pm 0,8 / 4,0 \\
(1-5)\end{array}$ \\
\hline $\begin{array}{l}\text { Valor de } \mathrm{p}^{\mathrm{a}} \\
\text { Torta de Soja }\end{array}$ & - & 0,147 & 0,038 & 0,044 & 0,044 & 0,002 \\
\hline Onívoros & $\begin{array}{l}1 \\
2 \\
0\end{array}$ & $\begin{array}{l}4,1 \pm 0,8 / 4,00 \\
(2-5)\end{array}$ & $\begin{array}{l}3,5 \pm 1,1 / 4,00 \\
(1-5)\end{array}$ & $\begin{array}{l}3,7 \pm 1,1 / 4,00 \\
(1-5)\end{array}$ & $\begin{array}{l}3,6 \pm 1,1 / 4,00 \\
(1-5)\end{array}$ & $\begin{array}{l}3,9 \pm 0,8 / 4,00 \\
(2-5)\end{array}$ \\
\hline Vegetarianos & $\begin{array}{c}2 \\
5\end{array}$ & $\begin{array}{l}3,6 \pm 1,3 / 4,00 \\
(1-5)\end{array}$ & $\begin{array}{l}3,4 \pm 1,4 / 3,00 \\
(1-5)\end{array}$ & $\begin{array}{l}3,6 \pm 1,3 / 4,00 \\
(1-5)\end{array}$ & $\begin{array}{l}3,7 \pm 1,2 / 4,00 \\
(1-5)\end{array}$ & $\begin{array}{l}3,8 \pm 1,2 / 4,00 \\
(1-5)\end{array}$ \\
\hline Valor de $\mathrm{p}^{\mathrm{a}}$ & - & 0,127 & 0,904 & 0,667 & 0,626 & 0,860 \\
\hline
\end{tabular}

Med: Média; DP: Desvio-padrão; Md: Mediana. n: Número de entrevistados; PTS: Proteína Texturizada de Soja; a: Teste de Mann-Whitney. O número de respondentes variou em função dos dias de coleta de dados. Fonte: Autores.

Verificou-se que entre os vegetarianos a preparação com maior índice de aceitação foi o Escondidinho de Cará com Proteína Texturizada de Soja (82,4\%), enquanto que entre os onívoros foi o Quibe Vegetariano (83,6\%). A Torta de Soja foi a preparação menos aceita por vegetarianos e onívoros $(72,2 \%$ e $77,7 \%$ respectivamente). 
Tabela 4 - Índice de aceitação das preparações vegetarianas fornecidas nos campi de um restaurante universitário, de acordo com a adesão à prática alimentar. Vitória - ES, 2019.

\begin{tabular}{|c|c|c|c|c|c|}
\hline $\begin{array}{l}\text { Preparação } \\
\text { Vegetariana }\end{array}$ & $\begin{array}{l}\text { IA Sabor } \\
(\%)\end{array}$ & $\begin{array}{l}\text { IA Aroma } \\
(\%)\end{array}$ & $\begin{array}{l}\text { IA Textura } \\
(\%)\end{array}$ & $\begin{array}{l}\text { IA Aparência } \\
(\%)\end{array}$ & $\begin{array}{l}\text { IA Aceitação } \\
\text { Geral } \\
(\%)\end{array}$ \\
\hline \multicolumn{6}{|c|}{$\begin{array}{l}\text { Escondidinho de Cará } \\
\text { com PTS }\end{array}$} \\
\hline Onívoros & 79,6 & 68,2 & 77,3 & 80,3 & 79,4 \\
\hline Vegetarianos & 88,4 & 75,3 & 78,9 & 73,4 & 82,4 \\
\hline \multicolumn{6}{|l|}{ Falafel } \\
\hline Onívoros & 81,4 & 70,3 & 75,3 & 71,5 & 79,3 \\
\hline Vegetarianos & 72,0 & 65,5 & 70,5 & 75,0 & 75,5 \\
\hline \multicolumn{6}{|c|}{ Polenta com Lentilha } \\
\hline Onívoros & 84,9 & 70,7 & 90,3 & 82,7 & 82,5 \\
\hline Vegetarianos & 75,5 & 70,0 & 76,7 & 76,7 & 77,5 \\
\hline \multicolumn{6}{|c|}{ Quibe Vegetariano } \\
\hline Onívoros & 83,9 & 74,5 & 76,7 & 81,6 & 83,6 \\
\hline Vegetarianos & 80,4 & 70,7 & 71,7 & 78,1 & 77,7 \\
\hline \multicolumn{6}{|l|}{ Torta de Soja } \\
\hline Onívoros & 80,3 & 71,8 & 74,3 & 67,4 & 77,7 \\
\hline Vegetarianos & 74,1 & 65,2 & 62,0 & 64,1 & 72,7 \\
\hline
\end{tabular}

IA: Índice de Aceitabilidade; PTS: Proteína Texturizada de Soja. Fonte: Autores.

Quanto ao resto-ingestão por cliente, no campus 1 verificou-se que a média \pm DP foi de $31,8 \pm 4,7 \mathrm{~g}$, variando de 21,0 a

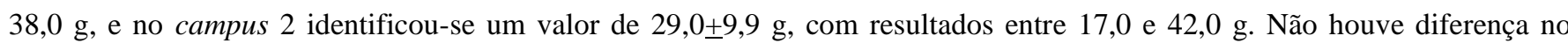
quantitativo de resto-ingestão/cliente entre os dois campi $(\mathrm{p}=0,59)$. Apesar de ser classificado como inadequado na maioria dos dias avaliados, especialmente para o campus 1, não foi encontrada associação entre a adequação do resto-ingestão e os campi $(\mathrm{p}=0,20)($ Tabela 5).

Tabela 5 - Quantificação e classificação do resto - ingestão por cliente em restaurantes universitários, segundo campus. Vitória - ES, 2019.

\begin{tabular}{lllll}
\hline Informações & Total & Campus 1 & Campus 2 & Valor de p \\
\hline Resto - ingestão médio por cliente (g) & & & & \\
Média \pm DP & $30,8 \pm 6,8$ & $31,8 \pm 4,7$ & $29,0 \pm 9,9$ & \\
Mínimo & 17,0 & 21,0 & 17,0 & $0,59^{\mathrm{a}}$ \\
Máximo & 42,0 & 38,0 & 42,0 & \\
Classificação do resto - ingestão per capita (\%) & & & \\
Adequado & 35,3 & 27,3 & 50,0 & \\
Inadequado & 64,7 & 72,7 & 50,0 & $0,20^{\mathrm{b}}$ \\
\hline
\end{tabular}

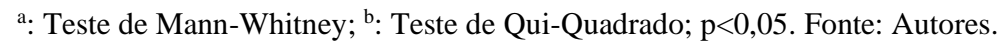

\section{Discussão}

No presente estudo foi identificado elevado desperdício de alimentos por parte dos clientes, fato este que pode ser indicativo da baixa satisfação com o cardápio. Em contraste, verificou-se que as preparações vegetarianas apresentaram notas altas para todos os atributos (sabor, aroma, textura, aparência e aceitação geral) e índice de aceitabilidade satisfatório (>70\%) (Dutcosky, 1996, Dutcosky, 2006), tanto para os onívoros, quanto para os vegetarianos.

Como esperado, a frequência de consumo da preparação vegetariana foi maior entre os que se aderiam a este estilo de vida. Nota-se que o atributo que obteve maior IA entre as preparações avaliadas foi o sabor e o que obteve menor IA foi o aroma. Entretanto, em um estudo que analisou a relação entre o cardápio e o desperdício de um restaurante universitário de Campinas - SP, $40 \%$ das pessoas entrevistadas relataram que o fator mais determinante para aceitabilidade e desperdício é o 
sabor (Almeida, Brito, Lakatos, \& Montemor, 2008). Em contraste, outro estudo realizado na Universidade Estadual do Rio de Janeiro, os maiores problemas relatados em uma preparação com base de proteína vegetal foi a textura (Nagagata et al. 2020).

Considerando que os hábitos alimentares desenvolvidos durante a vida universitária são heterogêneos, a aceitabilidade dos usuários frente às preparações vegetarianas sofre influências de fatores como crenças religiosas e/ou culturais, educação familiar, intolerâncias e alergias, gosto pessoal por um alimento ou ingrediente da preparação (França, 2017). Diante disso, torna-se imprescindível avaliar a satisfação dos clientes com os cardápios, além de averiguar suas preferências a fim de aumentar a aceitabilidade. Ademais, após essa avaliação do cardápio, o treinamento dos funcionários, elaboração de ficha técnica de preparo e sua replicação padronizada são fatores fundamentais para que as preparações e cardápios sejam bem aceitos pelos clientes em todos os atributos como os pesquisados no presente estudo (Oliveira, Santos, Nascimento, \& Santos, 2017).

Acredita-se que seja complexo satisfazer, em maior grau, um público que não é vegetariano e não está acostumado com as particularidades sensoriais desses alimentos comumente utilizados nas preparações vegetarianas em restaurantes universitários como, por exemplo, a Proteína Texturizada de Soja (Narciso, Rego, Meneguessi, \& Iahnn, 2017). Entretanto, a aceitação geral neste estudo foi maior entre onívoros do que entre vegetarianos em quatro de cinco preparações avaliadas.

O rejeito alimentar, como um indicador de satisfação do cardápio, pode ser influenciado por diversos fatores como, por exemplo, a qualidade da refeição, temperatura da preparação, apetite do cliente, tamanho da porção servida e clima (Soares, Pereira, Gomes, \& Oliveira, 2018). Embora o ideal, na literatura, de RI por pessoa seja inferior a 15g, considera-se valores aceitáveis aqueles que ficam entre 15 e $45 \mathrm{~g}$ por cliente (Vaz, 2006). Sendo assim, torna-se necessário que o gestor e a equipe da UAN monitorem os indicadores de desperdício por um determinado período de tempo e estabeleça o seu parâmetro próprio (Abreu, Spinelli, \& Pinto, 2011).

Nascimento et al. (2020) evidenciaram valores de RI/cliente entre 27 e $40 \mathrm{~g}$ por pessoa, em um RU localizado em Santa Teresa - ES. O valor máximo evidenciado no estudo supracitado se aproxima da quantidade de RI/cliente do campus 2, de 42,1 g. Massarollo, Fagundes e Prieto (2019) avaliando o resto-ingestão de um RU no Paraná, verificaram que a média de $\mathrm{RI} /$ cliente foi de 30g, próxima da média evidenciada nesta pesquisa. Em contrapartida, a avaliação do desperdício de alimentos em um RU do Piauí resultou um peso médio de RI/cliente de 167g (Soares, Pereira, Gomes, \& Oliveira, 2018) um valor $556,7 \%$ acima do encontrado neste estudo.

O desperdício de alimentos é foco de discussão mundial e de criação de políticas públicas, pois atinge cerca de 1,3 bilhão de toneladas por ano (Food and Agriculture Organization, 2013). No Brasil, um dos principais fatores responsáveis por esse desperdício é a falta de conscientização social sobre a quantidade de resíduos produzidos que poderiam ser reaproveitados (Borges, Borges, \& Lopes, 2016). Portanto, os valores encontrados acima dos recomendados pela literatura refletem a importância de se conhecer os fatores relacionados a esse desperdício em UAN como, por exemplo, o cardápio que não agrada a clientela e o porcionamento das preparações (Brito \& Oliveira, 2017).

O presente trabalho compreendeu um público predominantemente jovem e estudante. Em um estudo que avaliou a qualidade de vida da população vegetariana no Brasil o maior público identificado também foi de jovens entre 18 e 24 anos de idade $(28,2 \%)$ (Hargreaves, Nakano, \& Zandonadi, 2020). Por se tratar de um ambiente de ensino, o espaço universitário é considerado propício para a realização de atividades voltadas para a promoção de hábitos alimentares saudáveis, práticas educativas e a difusão de temáticas de saúde, sociais e ambientais, como a de sustentabilidade (Moreira, Pafiadache, \& Loose, 2015). Além disso, vale mencionar que um dos motivos mais comuns na adesão à pratica vegetariana está relacionado às razões ambientais, sendo a preocupação com a sustentabilidade uma temática importante em relação ao futuro dessa prática alimentar (Leitzmann, 2014). Portanto, nessa mesma perspectiva, o vegetarianismo também representa uma prática sustentável. 
Neste estudo, a alimentação vegetariana foi mencionada por um quinto dos participantes e a modalidade mais adotada foi a ovolactovegetariana. Ressalta-se que apesar do crescimento de diferentes modalidades, incluindo o veganismo, a dieta ovolactovegetariana tem se mostrado frequente entre os universitários (Aplleby, Thorogood, Mann, \& Key, 1999), sendo este tipo comum também aos que estão em transição da dieta onívora para ao vegetarianismo estrito ou veganismo.

Um estudo realizado em 2018 com universitários do Sul do Brasil também investigou os fatores associados à prática alimentar e encontrou, assim como nesta pesquisa, predominância de onívoros $(93,6 \%)$ e de ovolactovegetarianos $(5,4 \%)$. Além disso, a prática vegetariana também se mostrou associada ao sexo feminino (Oliveira, Santos, Nascimento, \& Santos, 2017). Outros estudos também apontam que o vegetarianismo é mais prevalente entre mulheres (Spencer, Elon, Frank, 2007, Clarys et al. 2014, Sociedade Vegetariana Brasileira, 2016, Hargreaves et al. 2020).

Rezende, Godinho, Souza e Ferreira (2015) ao analisar o perfil de universitários vegetarianos, encontraram que a idade média dos respondentes foi de 23 anos, próxima à obtida no presente estudo. Ressalta-se que pela amostra da pesquisa ser predominantemente de estudante, observa-se uma idade menor comparada aos estudos conduzidos com a população vegetariana geral, que no Brasil se concentra entre 55 anos ou mais (Brasil, 2018), enquanto nos Estados Unidos a faixa etária predominante dos adeptos está entre 35 e 44 anos (Stahler, 2019).

\section{Considerações Finais}

A satisfação do cardápio, avaliada pelo desperdício de alimentos, se mostrou baixa nos locais avaliados. Quanto à aceitabilidade das preparações vegetarianas, observou-se que embora o índice específico para cada atributo (sabor, aroma, textura, aparência e aceitação geral) tenha variado, a aceitabilidade foi satisfatória para a maioria das preparações. Vegetarianos e onívoros atribuíram notas semelhantes para as preparações, com exceção do Quibe Vegetariano que foi melhor avaliado pelos onívoros.

Diante do exposto, sugere-se a realização periódica de pesquisas de satisfação com os clientes para identificar mudanças em seu hábito e preferências alimentares e direcionar o planejamento adequado do processo de produção de refeições em UAN, além de contribuir para a diminuição no desperdício. Ainda se fazem necessários, o monitoramento contínuo dos indicadores de desperdício e a realização de campanhas de conscientização dos clientes para a promoção de boas práticas ambientais.

Os registros presentes nesse estudo contribuem para a implantação de medidas corretivas em alimentação coletiva e podem, ainda, servir como subsídio para propostas de intervenção e instigar a realização de outros estudos relacionados à prática vegetariana em restaurante universitário.

\section{Referências}

Abreu, E. S., Spinelli, M. G. N. \& Pinto, M. A. S. (2011). Gestão de unidades de alimentação e nutrição: um modo de fazer. (4a ed). Metha.

Allés, B., Baudry, J., Méjean, C., Touvier, M., Péneau, S., Hercberg, S. \& Kesse-Guyot, E. (2017). Comparison of sociodemographic and nutritional characteristics between self-reported vegetarians, vegans, and meat-eaters from the NutriNet-Santé study. Nutrients, 9 (9): 1023.

Almeida, T. D., Brito Neto, J. L., Lakatos, M. \& Montemor, M. (2008). Relação entre o cardápio do restaurante universitário e desperdício. Revista Ciências do Ambiente On-Line 2008, 4 (1): 1-6.

Appleby, P. N., Thorogood, M., Mann, J. I. \& Key, T. J. (1999). The Oxford vegetarian study: an overview. American Journal of Clinical Nutrition, 70 (3): $525 \mathrm{~s}-531$.

Associação Brasileira das Empresas de Refeições Coletivas (2008). História e Mercado. https://www.aberc.com.br/conteudo.asp?IDMenu=18.

Bacalhau, S. P. O., Silva, M. S. S., Alves, J. F. C., Lopes, F. C. L., Gomes, A. A., Ramos, G. M. S. \& Menezes, J. S. O. (2016). Campanha prato limpo: evitando o desperdício de alimentos no refeitório do IFPE Campus Belo Jardim. Revista Conexões, Ciência e Tecnologia, 10 (1): $44-50$.

Barros, K. S., Bierhals, I. O. \& Assunção, M. C. F. (2020). Vegetarianismo entre ingressantes de uma universidade pública no sul do Brasil, 2018. Epidemiologia e Serviços de Saúde, 29 (4):20. 
Benvindo, J. L. S., Pinto, A. M. S. \& Bandoni, D. A. (2017). Qualidade nutricional de cardápios planejados para restaurantes universitários de universidades federais do Brasil. Demetra, 12 (2): 447-464.

Borges, V. M. B., Borges Neta, M. V. \& Lopes, J. N. S. (2016). Controle de sobras e resto-ingesta em restaurante self-service em Juazeiro do Norte - CE. Revista e-ciência, 4 (2): 63-69.

Brasil. Conselho Federal de Nutricionistas (CFN). (2018). Resolução CFN $n^{\circ} 600$, de 25 de fevereiro de 2018. Dispõe sobre a definição das áreas de atuação do nutricionista e suas atribuições, indica parâmetros numéricos mínimos de referência, por área de atuação, para a efetividade dos serviços prestados à sociedade e dá outras providências. https://www.cfn.org.br/wp-content/uploads/resolucoes/Res_600_2018.htm.

Brasil. Instituto Brasileiro De Opinião Pública E Estatística. Pesquisa de opinião pública sobre o vegetarianismo. (2018). 2021, de https://www.svb.org.br/images/Documentos/JOB_0416_VEGETARIANISMO.pdf>.

Brasil. Programa Nacional de Assistência Estudantil - PNAES. (2010). Decreto n ${ }^{o} 7234$, de 19 de julho de 2010. Dispõe sobre o programa nacional de assistência estudantil. http://www.planalto.gov.br/ccivil_03/_ato2007-2010/2010/decreto/d7234.htm.

Brito, A. D. P. \& Oliveira, F. R. G. (2017). Desperdício alimentar: conscientização dos comensais de um serviço hospitalar de alimentação e nutrição. Revista Brasileira de Ciências da Saúde, 24 (2): 61-64.

Clarys, P., Deliens, T., Huybrechts, I., Deriemaeker, P., Vanaelst, B., Keyzer, W., Hebbelinck, M. \& Mullie, P. (2014). Comparison of nutritional quality of the vegan, vegetarian, semi-vegetarian, pesco-vegetarian and omnivorous diet. Nutrients, 6 (3): 1318-1332.

Craig, W. J. \& Mangels, A. R. (2009). Position of the American Dietetic Association: vegetarian diets. Journal of the American Dietetic Association, 109 (7): 1266-1282.

Dutcosky, S. D. (1996). Análise sensorial de alimentos. Champagnat.

Dutcosky, S. D. (2011). Análise Sensorial de Alimentos. (3a ed.) Champagnat.

Food and Agriculture Organization (FAO). (2013). Food wasteg foodprint: impacts on natural resouces. http://www.fao.org/publications/card/en/c/000d4a327304-5785-a2f1-f64c6de8e7a2/

França, J. F. M. (2017). Refeições vegan em ambiente escolar: Lei $n^{\circ}{ }^{\circ} 11 / 2017$ e as suas implicações em Unidades de Alimentação e Nutrição. Faculdade de Ciências da Nutrição e Alimentação da Universidade do Porto. https://repositorioaberto.up.pt/bitstream/10216/107054/2/210281.pdf.

Hargreaves, S. M., Nakano, E. Y. \& Zandonadi, R. P. (2020). Brazilian Vegetarian Population - Influence of Type of Diet, Motivation and Sociodemographic Variables on Quality of Life Measured by Specific Tool (VEGQOL). Nutrients, 12 (5): 1-22.

Instituto Brasileiro de Geografia e Estatística. (2019). Pesquisa de orçamentos familiares 2017-2019: análise do consumo alimentar pessoal no Brasil. https://biblioteca.ibge.gov.br/visualizacao/livros/liv101670.pdf

Leitzmann, C. (2014). Vegetarian nutrition: past, present, future. American Journal of Clinical Nutrition, 100 (1): 496-502.

Massarollo, M. D., Fagundes, E. M. \& Prieto, L. M. (2019). Avaliação do resto-ingesta em um restaurante universitário do município de Francisco Beltrão PR. Revista Brasileira de Obesidade, Nutrição e Emagrecimento, 13 (81): 703-707.

Minim, V. P. R. (2006). Análise sensorial: estudos com consumidores. Editora.

Moreira, F. J., Junior, Pafiadache, C., Loose, L. H., Piaia, R., Scher, V. T., Peripolli, A. \& Palm, B. (2015). Satisfação dos Usuários Do Restaurante Universitário Da Universidade Federal De Santa Maria: Uma Análise Descritiva. Revista Sociais e Humanas, 28 (2): 83-108.

Nagagata, B. A., Carvalho, C. F., Santos, L. P., Santana, I., Freitas, S. M. L. \& Guimarães, R. R. Development of vegan burgers: a study with consumers and market research. Research, Society and Development, 9 (7): 1-5.

Narciso, U. R., Rego, A. S., Meneguessi, B., Iahnn, S. R. (2017). Avaliação de aceitabilidade e desenvolvimento de preparações a base de proteína de soja para consumidores do Restaurante Universitário da Universidade Federal da Grande Dourados. http://eventos.ufgd.edu.br/enepex/anais/arquivos/2075.pdf

Nascimento, M. C. F., Ferreira, E. P., Silva, B. G., Ferreira, Y. P., Fabris, P., Kruger, F. C., Pantaleão, F. S. \& Nascimento, M. V. F. (2020). Índice de aceitabilidade e resto ingesta em unidade de alimentação e nutrição: estudo de caso no Ifes campus Santa Teresa. Brazil. Brazilian Journal of Health Review, 3 (2): $1868-1880$

Oliveira, M. C., Santos, C. R. B., Nascimento, H. S. \& Santos, I. P. G. (2017). Ambientes alimentares universitários: percepções de estudantes de Nutrição de uma instituição de ensino superior. Demetra, 12 (2): 431-445.

Rezende, E. T., Godinho, S. E., Souza, A. C. N. \& Ferreira, L. G. (2015). Ingestão proteica e necessidades nutricionais de universitários vegetarianos. Revista de Atenção à Saúde, 13 (44): 52-57.

Soares, T. C., Pereira, A. C. S., Gomes, S. A. B. \& Oliveira, E. S. (2018). Avaliação do desperdício de alimentos servidos no horário do almoço em Restaurante Universitário no estado do Piauí, Brasil. Revista Brasileira de Higiene e Sanidade Animal, 12 (3): 271-279.

Sociedade Vegetariana Brasileira. (2016). Mercado vegetariano. Recuperado em 26 de março de 2021, de https://www.svb.org.br/vegetarianismo1/mercadovegetariano>.

Spencer, E. H., Elon, L. K. \& Frank, E. (2007). Personal and professional correlates of US medical students' vegetarianism. Journal of American Dietetic Association, 107 (1): 72-78. 
Research, Society and Development, v. 10, n. 12, e44101220109, 2021

(CC BY 4.0) | ISSN 2525-3409 | DOI: http://dx.doi.org/10.33448/rsd-v10i12.20109

Stahler, C. (2019). How many people are vegan? How many eat vegan when eating out? Asks the vegetarian resource group. https://www.vrg.org/nutshell/Polls/2019_adults_veg.htm.

Tonstad, S., Stewart, K., Oda, K., Batech, M., Hering, R. P. \& Fraser, G. E. (2013). Vegetarian diets and incidence of diabetes in the Adventist Health Study-2. Nutrition, Metabolism and Cardiovascular Diseases, 23 (4): 292-299.

Vaz, C. S. (2006). Restaurantes: controlando custos e aumentando lucros. Metha.

Viana, R. M. \& Ferreira, L. C. (2017). Avaliação do desperdício de alimentos em unidade de alimentação e nutrição cidade de Januária, MG. Higiene Alimentar, 31 (266): 22-26. 